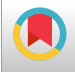

\title{
Emergency Management Strategies for Children with Rheumatic Disease During COVID-19 Epidemic Outside Hubei in China
}

\author{
Xinyi Wei ${ }^{\# 1}$, Xiaoke Sun ${ }^{\# 1}$, Zhi Chen ${ }^{1}$, Xiao $\mathrm{Du}^{1}{ }^{1}$, Jing $\mathrm{Li}^{2}{ }^{2}$, Omar Mechali ${ }^{3}$, Chun Jiang ${ }^{1,{ }^{*}}$ and Wei \\ Zhang $^{1, * *}$ \\ ${ }^{1}$ Pediatric Immunology and Rheumatology Department, School of Medicine, Chengdu Women's and Children's Central Hospital, University of Electronic Science and \\ Technology of China, Chengdu, China \\ ${ }^{2}$ Department of Nosocomial Infection Management, School of Medicine, Chengdu Women's and Children's Central Hospital, University of Electronic Science and Technology \\ of China, Chengdu, China \\ ${ }^{3}$ University of Electronic Science and Technology, Chengdu, China \\ "Corresponding author: Pediatric Immunology and Rheumatology Department, School of Medicine, Chengdu Women's and Children's Central Hospital, University of \\ Electronic Science and Technology of China, Chengdu, China. Email: 911070565@qq.com \\ ${ }^{* *}$ Corresponding author: Pediatric Immunology and Rheumatology Department, School of Medicine, Chengdu Women's and Children's Central Hospital, University of \\ Electronic Science and Technology of China, Chengdu, China. Email: gczhangwei@163.com \\ \# These authors are contributed equally as the first author.
}

Received 2020 June 14; Revised 2020 August 18; Accepted 2020 September 11.

\begin{abstract}
Background: Since December 2019, many patients with novel coronavirus pneumonia (NCP) have appeared in Wuhan, Hubei Province, China. The pneumonia was caused by a novel coronavirus named "coronavirus disease 2019, COVID-19" by the WHO on February 11, 2020. The COVID-19 outbreak has created a global public health crisis, and the challenge of combatting this pandemic and its impact is daunting. At present, vaccines and prophylactic therapies for COVID-19 are not available. What we can do now is to aggressively implement infection control measures to prevent the spread of COVID-19 via human-to-human transmission.

Objectives: The study aimed to summarize the effect of classification and division management methods on the prevention and control of COVID-19 in the Pediatric Immunology and Rheumatology Department.

Methods: Corresponding and targeted medical countermeasures for the prevention and control of COVID-19 from the aspects of personnel, ward, and process management were retrospectively analyzed.

Results: From January 16, 2020, to July 31, 2020, there were 11,000 outpatients with rheumatic disease visiting our hospital and 565 children with rheumatic diseases hospitalized in our department. All the patients received timely and reasonable treatment. None of the patients, their caregivers, or medical staff acquired COVID-19 infection in our department, and no suspected COVID-19 case was confirmed in our ward.

Conclusions: The model of classification and division management ensured the orderly and safe treatment of children with rheumatic disease during the epidemic prevention and control period, and protected the medical staff from occupational injuries at work, and brought the pandemic under control.
\end{abstract}

Keywords: COVID-19, Children, Rheumatic Diseases, Prevention

\section{Background}

Since December 2019, many patients with novel coronavirus pneumonia (NCP) have emerged in Wuhan, Hubei Province, China. The rapid onset, strong infectivity, and rapid changes are considered as the characteristics of this disease. The WHO announced the NCP epidemic in China as a public health emergency of international concern on January 31, 2020 (1). Pneumonia caused by the novel coronavirus was named "coronavirus disease 2019, COVID-19" by the WHO on February 11, 2020 (2).

COVID-19 has been reported in 216 countries and re- gions around the world, with over 17.6 million afflicted cases and 680,000 confirmed deaths until July 31, 2020, which is still in an upward trend (3). According to the National Health Commission of the People's Republic of China and Health Commission of Hubei Province, 84,337 afflicted cases and 4,634 deaths have been confirmed in China, and the corresponding data were 16,202 cases and 122 deaths among the general population outside Hubei in China until July 31, 2020.

The COVID-19 outbreak has created a global public health crisis, and the challenge of combatting this pan-

Copyright ( ) 2020, International Journal of Infection. This is an open-access article distributed under the terms of the Creative Commons Attribution-NonCommercial 4.0 International License (http://creativecommons.org/licenses/by-nc/4.0/) which permits copy and redistribute the material just in noncommercial usages, provided the original work is properly cited. 
demic and its impact is daunting. Given the lack of safe and effective vaccines or proven treatments for COVID-19, our main strategy to combat the pandemic is to aggressively implement infection control measures to prevent the spread of COVID-19 via human-to-human transmission.

\section{Objectives}

It has been reported that COVID-19 is an asymptomatic infection or presents with mild and nonspecific symptoms among infants and children (4). Children with rheumatic diseases may experience more severe symptoms when suffering from COVID-19 infection (5). It is particularly important to take measures to bring the pandemic under control and protect children with rheumatic disease, their caregivers, and medical staff from COVID-19 infection.

\section{Methods}

Measures from the aspects of personnel, ward, and process management were taken for protecting children with rheumatic disease (outpatient or inpatient), caregivers, and medical staff in our department from COVID-19 infection, and the effects of these measures were retrospectively analyzed.

\subsection{Management of Personnel}

\subsubsection{Children with Rheumatic Disease and Their Caregivers}

We established a network system for the management of children with rheumatic disease. Through this system, medical staff could timely understand the condition of children, provide them with disease-related knowledge, and spread health education for preventing COVID-19, including 'when and how to wash hands', 'how to wear a mask', 'coughing etiquette', etc.

The children with stable conditions could be followed up at home through the online consultation platform of the hospital's internet network, and the drugs could be sent home through the internet of things (IoT) to reduce the unnecessary commuting from home to the hospital during the epidemic period;

In the case of severe conditions, going to the hospital for timely examination and treatment was recommended;

When coming to the hospital, the children and caregivers should take self-protection measures, wear masks, avoid public transportation, avoid touching the surface of objects in the public area, and avoid crowded places;
During hospitalization, patients and their caregivers should wander around as little as possible, and should not leave the ward for any purpose but examination;

It was stipulated that one child should be taken care of by one caregiver, and two fixed caregivers might be left to take care of children under three years old as appropriate, avoiding frequent replacement. The access control system should be implemented in the ward, and the children should enter and exit the ward via an admission certificate. The access card sent by the ward was the only certificate for caregivers to enter and exit the ward;

We checked the body temperature of caregivers three times a day and made records. If the body temperature of the caregiver was higher than $37.3^{\circ} \mathrm{C}$, he/she would be advised to go to the designated hospital for medical treatment and be replaced by another healthy caregiver. The nurse should make a good registration of the physical condition of the caregivers every day.

\subsubsection{Medical Staff}

Standard preventive measures were used by all medical staff in the hospital, focusing on the implementation of key measures such as the correct wearing of masks and hand hygiene.

Three levels of protection were implemented by medical staff during the epidemic. According to different work positions, medical staff took measures in strict accordance with protection requirements (Table 1 )

To avoid cross-infection, special training for medical staff on COVID-19 and hospital-acquired infection prevention was conducted online and as self-study. The staff was assessed after learning, and the learning effect was supervised regularly.

\subsection{Management of Ward}

The ward was divided into two areas: The general area and the observation area. Each area was managed by certain medical staff;

\subsubsection{General Area}

The space between two beds must be more than one meter, and the curtain between two beds must be pulled down. Medical staff in the general area needed to take grade I protection (Table 1). Air disinfection should be taken two times a day, each time for $30 \mathrm{~min}$. The floor of the ward should be dragged with chlorine-containing disinfectants $(1000 \mathrm{mg} / \mathrm{L})$. We also wiped the surfaces with chlorine-containing disinfectant $(1000 \mathrm{mg} / \mathrm{L})$ two times a 


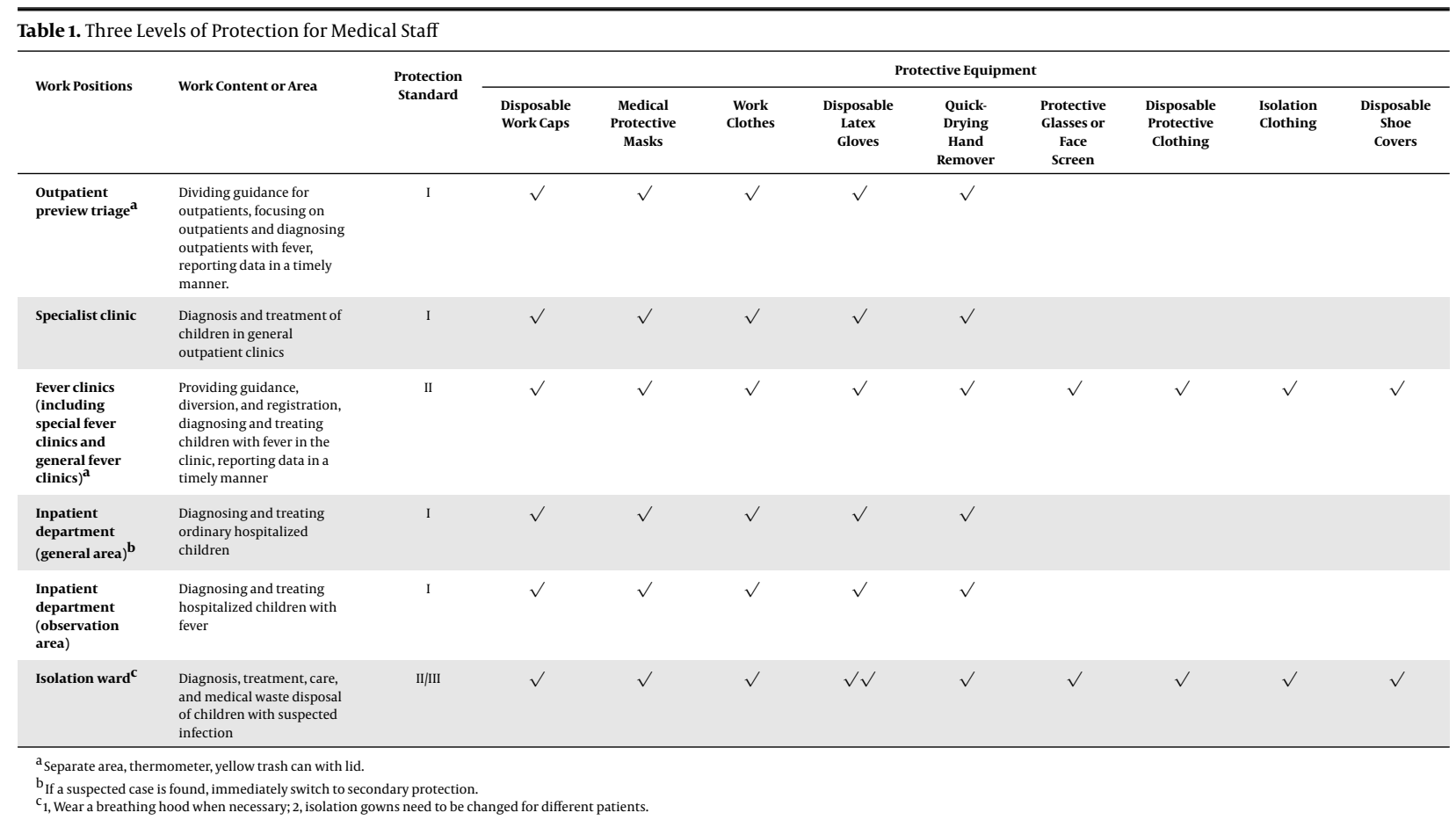

day, every time for more than $30 \mathrm{~min}$. Children in the general area would be discharged directly if the disease was cured. If fever occurred during hospitalization, the child would be transferred to the observation area for further treatment;

\subsubsection{Observation Area}

Every patient in the observation area stayed in a single ward. The medical staff in the observation area should take grade II protection. Air disinfection should be taken two times a day, each time for $30 \mathrm{~min}$. The floor of the ward should be dragged with chlorine-containing disinfectants $(2000 \mathrm{mg} / \mathrm{L})$. We also wiped the surfaces with chlorine containing-disinfectants $(2000 \mathrm{mg} / \mathrm{L})$ two times a day, each time for more than $30 \mathrm{~min}$. When the clinical symptoms of children in the observation area disappeared, and their body temperature remained normal for $48 \mathrm{~h}$ after treatment, they could be transferred to the general area until discharge;

\subsection{Management of Process}

\subsubsection{Outpatient and Hospitalization Procedures}

We strictly divided the hospital districts, and established special medical staff/patient passageways and action road maps for single entry and exit to avoid crossinfection;
All patients needed to be carefully asked if there was an epidemiological history (6). Single temperature entrance screenings were set up at the admission passages (for pedestrians and vehicles);

All patients or guardians must sign a commitment form about epidemic prevention, which included the cases of concealing epidemiological history and medical history; otherwise, the legal responsibility should be assumed;

A three-level triage system was implemented after admission: Nurses at the entrance of the outpatient building and triage nurse stations, and doctors at the consultation rooms. Children's body temperature was monitored, and epidemiological history was carefully asked again;

If the child had no fever or no epidemiological history, he/she was admitted to a specialist outpatient clinic or the general area of the ward for further treatment;

If the child had a fever, medical protective masks were immediately provided to the patient and guardians. If the children had an epidemiological history, patients should enter the special fever clinic. If the children did not have epidemiological history, patients should enter the general fever clinic. According to the doctor's examination, patients suspected of COVID-19 were transferred to an isolation area for treatment. If suspected COVID-19 was excluded, patients in need of further treatment would be ar- 
ranged to the observation area of the ward.

\subsubsection{Emergency Plan for Suspected COVID-19 Cases in the Ward}

When a suspected case of COVID-19 presented in the ward, the department director and the head nurse were immediately notified, and the hospital's first-level consultation team was called for help. The team consisted of specialists from our department and pediatric respiratory department. If the suspected COVID-19 case remained unconfirmed, the second-level consultation team was invited for further checks, consisting of multidisciplinary experts in the children's respiratory department, intensive care unit, emergency department, radiology department, and laboratory. If the suspected case was confirmed, the emergency response to suspected COVID-19 patients would be launched, and the patients should be transferred to the isolation ward (Figure 1).

\section{Results}

From January 16 to July 31, 2020, there were 11,000 outpatients with rheumatic disease visiting our hospital and 565 children with rheumatic disease hospitalized at our department. All of the patients received timely and reasonable treatment; these patients and their caregivers cooperated to take measures for the prevention and control of COVID-19 infection. The completion rate of tertiary protective measures for medical personnel in all positions was $100 \%$. None suffered from COVID-19 in our department, and no suspected COVID-19 case was confirmed in our ward.

\section{Discussion}

Based on the current epidemiological investigations and research results, the incubation period of the novel coronavirus is $1 \sim 14$ days (mostly $3 \sim 7$ days)(7). The main sources of infection are COVID-19 cases and asymptomatic virus carriers (8). By now, respiratory droplets and contact are regarded as the main routes of transmission, while aerosols and the fecal-oral route are still in controversy. Moreover, all people are generally susceptible (9), and children are less likely than adults to be infected with COVID19 (4). It is reported that children infected with COVID19 are mostly asymptomatic carriers, and the main potential causes of the spread and transmission of the disease in communities $(5,10)$. Providing safe and reasonable prevention strategies on COVID-19 needs to be considered all the time during the epidemic.
The abnormal immune response to the components of the body leads to tissue damage. Children with rheumatism disease need to extensively use glucocorticoids and immunosuppressants, sometimes in addition to biological agents and other drugs that control inflammation, which suppress immune responses. As a consequence, the defense of children with rheumatism disease to pathogens will significantly reduce, which may increase the risk of infection $(11,12)$. Therefore, it is particularly important to take measures to prevent COVID-19 infection and establish certain clinical management policies for children with rheumatic disease.

In this note, we summarized our outbreak fighting procedure and management process against the spread of COVID-19 infection during the epidemic period. As pediatricians in rheumatism, the adopted working methodology has allowed us to establish an appropriate working flow in our medical facility. The above procedures have conclusively proven their effectiveness, as no infection rose among children, caregivers, or medical staff.

The outbreak of COVID-19 is spreading all over the world. The epidemic has affected and changed the treatment and hospitalization process of children with rheumatism (13). This article is based on the concept of "scientific decision-making, humanistic service" to optimize the clinical work for children with rheumatism and improve the quality of these children's life during the COVID19 epidemic outside Hubei in China.

\section{Footnotes}

Authors' Contribution: Xinyi Wei and Xiaoke Sun contributed to the literature search, and writing and editing of the manuscript. Xiao Du contributed to literature search. Zhi Chen contributed to overall content and literature search, intellectual content, and drafting. Chun Jiang and Wei Zhang contributed to revising the manuscript. Jing Li and Omar Mechali contributed to providing consultation during the study.

Conflict of Interests: The authors declare no potential conflict of interest for the research, authorship, or publication of this article.

Funding/Support: This study was financially supported by the Science Foundation of Health Commission of Sichuan Province (grant no.: 20ZD019). 


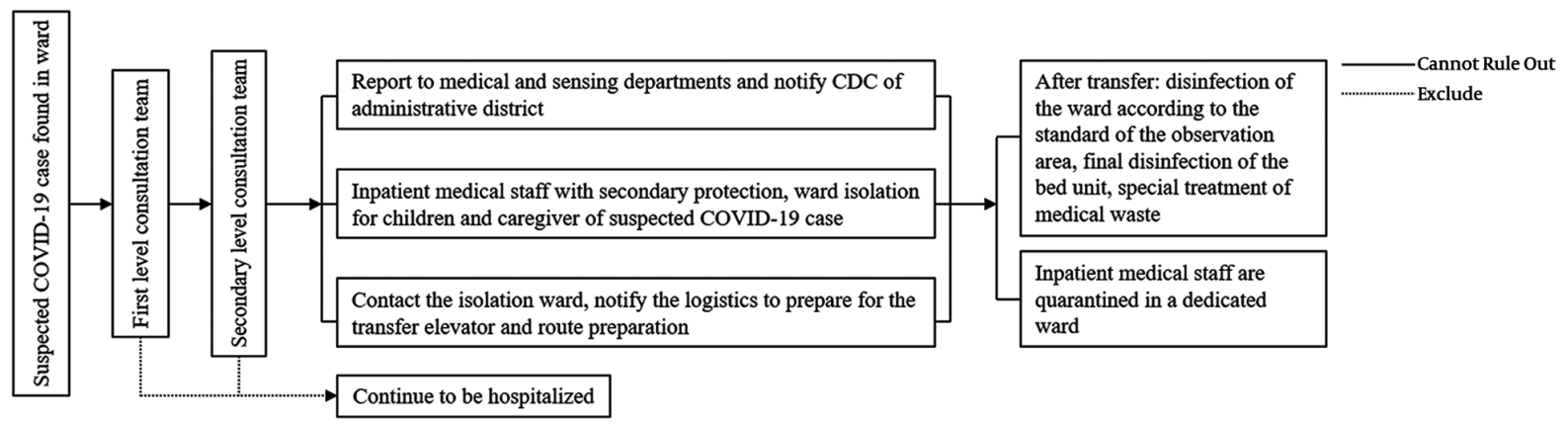

Figure 1. The emergency treatment process for suspected COVID-19

\section{References}

1. World Health Organization. Surveillance case definitions for human infection with novel coronavirus(nCoV). 2020, [cited 2020 Jan 31]. Available from: https://www.who.int/internal-publicationsdetail/surveillance-case-definitions-forhuman-infectionwithnovel-coronavirus-(ncov).

2. World Health Organization. Director-General's remarks at the media briefing on COVID-19. 2020, [cited 2020 Feb 11]. Available from: https://www.who.int/dg/speeches/detail/who-directorgeneral-sremarks-at-the-media-briefing-on-2019-ncov-on-11-february2020.

3. World Health Organisation. Coronavirus disease (COVID-19) situation reports. 2020, [cited 2020 Jul 31]. Available from: https://www.who.int/ emergencies/diseases/novel-coronavirus-2019.

4. Yang P, Liu P, Li D, Zhao D. Corona Virus Disease 2019, a growing threat to children? J Infect. 2020;80(6):671-93. doi: 10.1016/j.jinf.2020.02.024. [PubMed: 32142929]. [PubMed Central: PMC7125808].

5. Seyedi SJ, Shojaeian R, Hiradfar M, Mohammadipour A, Alamdaran SA. Coronavirus Disease 2019 (COVID-19) Outbreak in Pediatrics and the Role of Pediatricians: A Systematic Review. Iran J Pediatr. 2020;30(2). doi: $10.5812 /$ ijp.102784.

6. Society of Pediatrics CMA, Editorial Board CJOP. [Recommendations for the diagnosis, prevention and control of the 2019 novel coronavirus infection in children (first interim edition)]. Zhonghua Er Ke Za Zhi. 2020;58(3):169-74. Chinese. doi: 10.3760/cma.j.issn.05781310.2020.03.001. [PubMed: 32135584].
7. Li X, Zai J, Wang X, Li Y. Potential of large "first generation" humanto-human transmission of 2019-nCoV. J Med Virol. 2020;92(4):44854. doi: 10.1002/jmv.25693. [PubMed: 31997390]. [PubMed Central: PMC7166825].

8. Rothe C, Schunk M, Sothmann P, Bretzel G, Froeschl G, Wallrauch C, et al. Transmission of 2019-nCoV Infection from an Asymptomatic Contact in Germany. N Engl J Med. 2020;382(10):970-1. doi: 10.1056/NEJMc2001468. [PubMed: 32003551]. [PubMed Central: PMC7120970].

9. Phan LT, Nguyen TV, Luong QC, Nguyen TV, Nguyen HT, Le HQ, et al. Importation and Human-to-Human Transmission of a Novel Coronavirus in Vietnam. N Engl J Med. 2020;382(9):872-4. doi: 10.1056/NEJMc2001272. [PubMed: 31991079]. [PubMed Central: PMC7121428].

10. Mission W. Report of the who-china joint mission on coronavirus disease2019 (covid-19). Geneva: WHO; 2020.

11. Sengler C. [New therapy approaches, better outcomes? : Results from inception cohorts for patients with juvenile idiopathic arthritis]. $Z$ Rheumatol. 2019;78(7):610-9. German. doi: 10.1007/s00393-019-0648-1. [PubMed: 31127369].

12. Horneff G. [New drugs for treatment of juvenile idiopathic arthritis]. Z Rheumatol. 2019;78(7):587-98. German. doi: 10.1007/s00393-0190646-3. [PubMed: 31098699].

13. Qian X, Ren R, Wang Y, Guo Y, Fang J, Wu ZD, et al. Fighting against the common enemy of COVID-19: a practice of building a community with a shared future for mankind. Infect Dis Poverty. 2020;9(1):34. doi: 10.1186/s40249-020-00650-1. [PubMed: 32264957]. [PubMed Central: PMC7137400]. 